\title{
Monitoring of the quality of the psychological component of teachers' activity of higher education institutions based on Google Forms
}

\author{
Olena Bondarchuk ${ }^{1, *}$, Valentyna Balakhtar ${ }^{2}$, and Kateryna Balakhtar $^{3}$ \\ ${ }^{1}$ University of Educational Management, Department of Management Psychology, Kyiv, Ukraine \\ ${ }^{2}$ Yuriy Fedkovych Chernivtsi National University, Department of Pedagogy and Social Work, Chernivtsi, Ukraine \\ ${ }^{3}$ Yuriy Fedkovych Chernivtsi University, Department of Communicative Linguistics and Translation, Chernivtsi, Ukraine
}

\begin{abstract}
The focus of the current research is on the quality of education as a multifaceted category, and the monitoring of the quality of education - as a purposeful and specially organized system of studying, assessment, analysis of data on the state of education of students. This study aims at exploring the nowadays conditions, and exactly the development of digital technologies. The need of using the electronic resources (Google services) has become more acute, which enables the creation of text documents, presentations, spreadsheets, forms, drawings and other documents. In order to provide the monitoring of the quality of the psychological component of the teachers' activity of higher education institutions the Google form was elaborated to obtain the information about the psychological state and satisfaction of the quality of educational services by the fellows of the educational process, their relationships, socio-psychological climate in higher education institutions and others, and respond in a timely manner to the social and educational changes.
\end{abstract}

\section{Introduction}

The quality of education as a key factor in the country's sustainable development plays an important role in creating a single European educational space. The highquality education itself is an important tool for the creating of the key competences that are significant for the present, the development both of the individual and society, the state as a whole, by ensuring the social and economic growth of the country.

The education right of each individual was regulated by Universal Declaration of Human Rights, and the adoption of the Incheon Declaration "Education-2030: Ensuring of Inclusive and Equitable Quality Education and Lifelong Learning for All" has been set out the fundamental principles for global education development by 2030 [1].

The quality of education is considered to be the heart of education for all [2, pp. 100-109], positively influencing on the changes in student learning (affective, cognitive, and psychomotor domains) and personal and professional potential [3, pp. 3-13]. Hence, the actualization of the monitoring problem of the quality of education in higher education institutions has been being extremely important presently.

The monitoring is an integral part of the management of the quality of education; a means of information diagnosing in the process of carrying the managerial decisions, analysing the educational activities, predicting the changes in the educational process etc. The alteration of the living conditions places the new demands on the quality of education, which requires the study and evaluation of education indicators, the monitoring of the quality of education as a major driver of personal growth and development, as well as the consideration of psychological factors of higher education quality that contribute to the development of the creative and safe environment in higher education institutions [4, pp. 8689].

The use of information and communication technologies in education creates new opportunities for individualization and differentiation of the educational process, allows you to easily and quickly adapt to the new requirements of the monitoring of the quality of education, ensuring the creation of an optimal environment for educational services, understanding of human behaviour in the social environment, life cycle development and interaction between biological, psychological, socio-structural, economic, political and cultural factors of the educational process $[5, \mathrm{pp} .93-$ 104].

The quality of educational process and the effectiveness of the knowledge provided are impossible without diagnostics and monitoring, among which the diagnostic forms are questioning and testing. From this perspective, Google Services attracts our attention as an innovative tool of cloud technology [6, pp. 79-83], which let us control, collect, summarize and analyse the information through questionnaires (surveys) using simple online forms (Google Form), view the

* Corresponding author: bei.07@ukr.net 
spreadsheets, and visualize the survey results in graphs and charts for further analysis.

Google Form is utterly convenient tool, the onlineservice for forming the feedback forms, tests and surveys in order to organize the remote interaction of participants and experts in the framework of the assessment of the quality of higher education.

\section{Literature review}

The qualities of education as a component of the "Education for All" program is a broad concept and without single interpretation defining its essence, content and components by now, moreover, but as Haddad \& Demsky [7], claim, it depends on the policy of the state. Quality education includes: students, educational environment, content, process and results.

The concept "quality" is constantly influenced by economy, politics, culture, so it should be flexible, capable of changing during the evolution of education and progress [8]. For instance, changeability, fairness, efficiency and quality are often used as synonyms [9].

The quality of education as a multifaceted category by its essence covers various aspects (philosophical, pedagogical, psychological, social, economic, etc.). Thus, the quality is understood as the normative level to which the product of enlightenment corresponds [10]; the level of achievement of certain goals and objectives of education, set of indicators characterizing various aspects of the educational process (content of education, forms and methods of teaching, etc.) [11] and others. The quality of education is about the value of education (with reference to its contribution to the learning process and its outcome) [12].

Control and monitoring of the quality of education, continuous monitoring of the educational process in order to identify its compliance with the desired result [13] and determining, where necessary, corrective and developmental measures, are the important tasks of monitoring aimed at the systematic collection, processing, storage and dissemination of information on the state of education, forecasting with reason of objective dynamics data and the main tendencies of its development and science-based recommendations making to take the managerial decisions according to the improving the efficiency of the education industry functioning [14]; the current adjustment of higher education institution's activity [15] etc.

The monitoring of the quality of the teachers' educational activities in higher education institutions in accordance with the public requirements and stakeholders' needs provides a purposeful and specially organized system of continuous (regular and planned) observation (study), measurement, evaluation, analysis of data on various aspects of teachers' professional activity, consequently, the forecasting, the development of science-based recommendations for timely management decisions due to the improving the quality of the educational process and results [16].

\section{The monitoring psychological component of the quality of higher education}

The Higher education quality as transformative process leads to a focus on psychological factors of quality of higher education which contribute to the development of a creative and safe educational environment [4]. The psychological component of the monitoring of the quality of education involves the providing the information about the psychological status and satisfaction with the quality of educational services of the participants of the educational process, their relationship, the sociopsychological climate in higher education institutions, etc.

The criteria of this component are: satisfaction of the requests and needs of the listeners, and indicators: 1) the level of satisfaction of the requests and needs of the listeners; 2) the relevance of the training content to the listeners' professional needs and the stakeholders' requests of as a whole; 3) the listeners' psychological status and level of satisfaction with the quality of educational services; 4) the nature of the relationship between the participants of the educational process and the level of satisfaction with them; 5) the sociopsychological climate in higher education institution as an indicator of the level of development of organizational culture $[16$, p. 6].

Therefore, it should be indicated, as the educational practice shows, the regular study and the assessment of the data is directly or indirectly carried out mainly by the first three indicators. Hereat, the analysis of data on the relevance of the quality of education to the requests not only of listeners, but also of stakeholders, as a whole, indicates the expediency of intensifying the processes of self-education, self-knowledge and self-development of education workers, improving their qualification. In particular, this is evidenced by the results of a large-scale study made by us during years 2014-2019 (about 1000 respondents from all regions of Ukraine who passed the advanced training at the Central Institute of Postgraduate Education of University of Educational Management) the attitude of the education institutions' employees to their psychological competence, by which the ambivalent character was revealed. In particular, assessing positively the results of the acquisition of psychological competence in higher education institutions, the respondents-educators mainly note its need to influence other participants of the educational process. Simply a small part of the listeners (less than $10 \%$ ) remarks the expediency of using the acquired knowledge for introspection, reflection of the process of their professional and personal development.

In regards to the indicators "the nature of the relationship between the participants of the educational process and the level of satisfaction with them"; as well as the "the socio-psychological climate as an indicator of the level of development of organizational culture", they were, despite all actuality, practically not monitored. Thus, the psychological component of the monitoring of the quality of education is not fully implemented and 
requires the special study, the procedure of which is greatly facilitated by the use of Google services.

\section{Google services in the educational activity}

It is well known that information and communication technologies are currently considered to be a wide range of digital technologies used to create, transmit, disseminate information and provide services (Internet, e-mail, software, etc.). Google services includes many concepts (infrastructure, platforms, software, data, etc.). The main function of Google services is to meet the users' needs required the remote processing and storage of data [17, pp. 293-306]. Google services is a fullfledged educational tool which enables most effectively to create the own online space and form a personal educational environment for teachers and students of higher education institutions. Google services is a flexible cost-effective model that can be easily and quickly adapted to new software requirements, supporting the standardization of such software and various applications, simplified maintenance through centralized updates. Moreover, they are supported by various devices (tablets, laptops, desktops, etc.) of teachers and students; can be used both in educational institutions and abroad; enable to save time and increase security through the remote control and maintenance, etc. [18, pp. 3-16].

Using Google services enables to develop the digital competency (the ability to consciously and critically use the digital society technologies) [19, pp. 316-336], as well as the information and communication competence as a person's ability to use information and communication technology in practice to meet individual needs and solve the socially significant, in particular, professional tasks in a subject area [20, pp. 226-245].

There are many types of Google services, but in the context of the problem under study we are interested in the service of the Google Drive cloud storage that can help organize the monitoring of the quality of education etc. In order to work with it, the Gmail should be created. The browser-based application is available for free to create any number of Google Forms (web pages) that host a form or a questionnaire. Google Form opens up an extremely wide field of opportunity and creates an opportunity to achieve the stated goal in a short time and collect answers to your questions.

\section{The monitoring procedure of the quality of the psychological component of the educational process}

Therewith, the improvement of the internal system of the ensuring with the monitoring of the quality of education in higher education institutions, we have compiled a series of express methods (based on Google Form) on the indicators "the nature of the relationship between the participants of the educational process and the level of satisfaction with them", "the socio-psychological climate in higher education institutions as an indicator of the level of development of organizational culture", etc. scientifically-based recommendations for further improvement of the quality of the educational process in the educational institution by the psychological component.

Google Forms permits: to make the questionnaire available to respondents as soon as it is created, upon it can be edited, meanwhile the questions can be opened and analysed; to embed it on a site page, distribute it through mobile networks, where it can be filled by potential respondents; the service automatically to generate a spreadsheet to collect and process the responses to the author of the form, and to display the results of the survey as a spreadsheet (or filtered list) which has all the features and capabilities of a regular Google chart; the service to make it possible to view the answers of all the respondents and separately each of them individually without a spreadsheet, but with the appropriate statistics in the diagrams and graphs data given in qualitative and percentage values formats; to summarize the answers in graphical and numerical format. We have created the form namely "The Quality of the Educational Process: the Psychological Component" containing four techniques. The first method due to the assessment the psychological atmosphere in the unit (team) proposes to choose the opposite by contents pairs of words (by A. Fiedler), which allows describing the nature of interpersonal relationships in the unit [21].

The respondent chooses the correct answer for him/her and puts a mark in each pair (1 to 7), thereby choosing closer to the right or left word, which indicates a more pronounced sign of interpersonal relationships in the unit, such as "agreement" or "disagreement", "satisfaction", "dissatisfaction", etc. (Fig. 1).

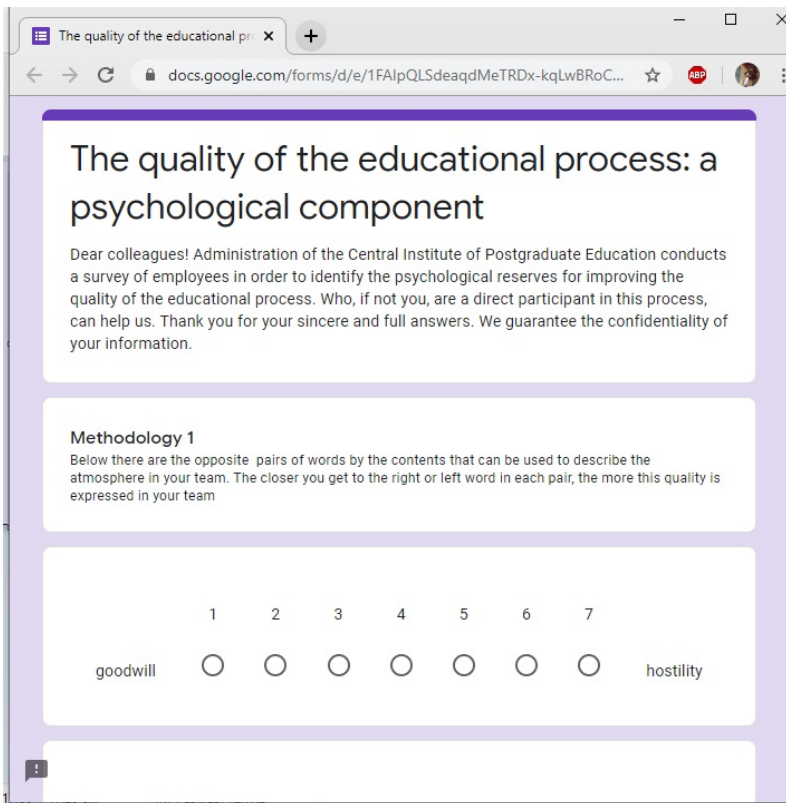

Fig. 1. The interface of Google forms for learning the quality of the educational process.

The screenshot highlights the title of the study "The Quality of the Educational Process: the Psychological 
Component", as well as the Method 1 instruction for the respondents to determine the psychological atmosphere in the team.

During the implementation of the second methodology for the diagnosis of psychological atmosphere in a small production group (authors V. Shpalynskyi, E. Shelest) [21], in the adaptation of O. Bondarchuk), on a 5-point scale, the respondents rate the degree of their favourable climate for the team (goodwill, trusting relationships, joy for success of colleagues, relations with management, adherence to rules in the team, etc.) (Fig. 2).

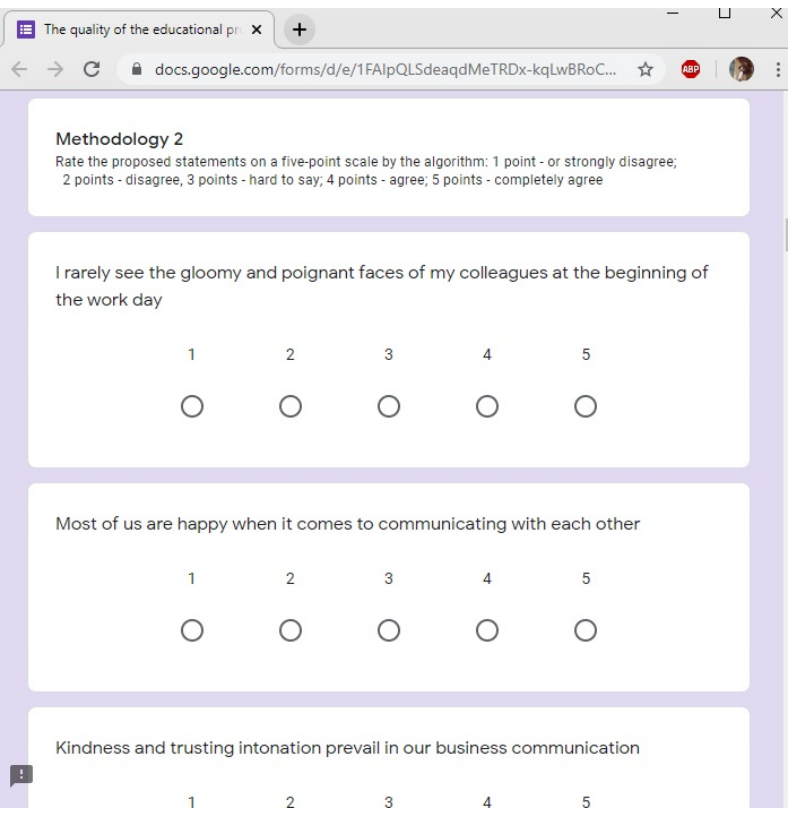

Fig. 2. The interface of Google forms for learning the psychological climate (Method 2).

Referring to Fig. 2, the Method 2 "Rate the proposed statements on the 5-point scale by the algorithm: 1 point - strongly disagree; 2 points - disagree; 3 points - hard to say; 4 points - agree; 5 points - completely agree" is shown on the previous screenshot.

Based on the answers of the respondents, the Google Form gives an opportunity to determine the level of favourable (high, above average, average, below average and unfavourable) psychological climate in the team.

Likewise, the Methods about learning the group cohesion (Sisor Index, adapted by O. Bondarchuk) and the psychological security of the educational environment, allowing determining the level of the psychological security in the educational environment (by I. Baeva, modified by O. Bondarchuk [6, 14-19]), (The Methods 3 and 4 respectively) were applied.

After filling in the Google Form, the respondent must click the button "Submit" (Fig. 3).

Referring to Fig. 3, the screenshot shows the option of choosing the answers for the employee's protection "From the unfriendly attitude of the administration" and "How satisfied are you with your life as a whole?". The "Submit" function is visible at the end.

After receiving the answers, we are able to review them, create a spreadsheet or chart where it is possible to examine the statistics for each question, analyse appropriately, evaluate etc. The results of the answers can be obtained in the form of the linear scale Fig. 4 or diagrams Fig. 5.

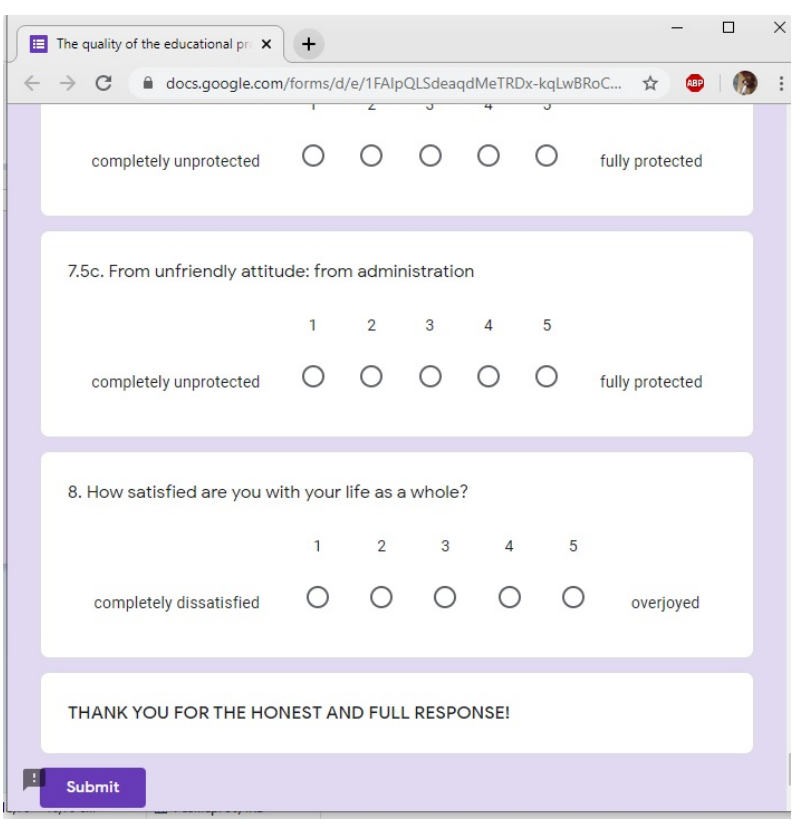

Fig. 3. The interface of the "Submit" function.

The example of the analysis of the answers in the form of the linear scale to the question "Would you go to another unit if this opportunity happened (without changing other conditions)?" and "What are the relationships between the members of your unit?" is given in Fig. 4.

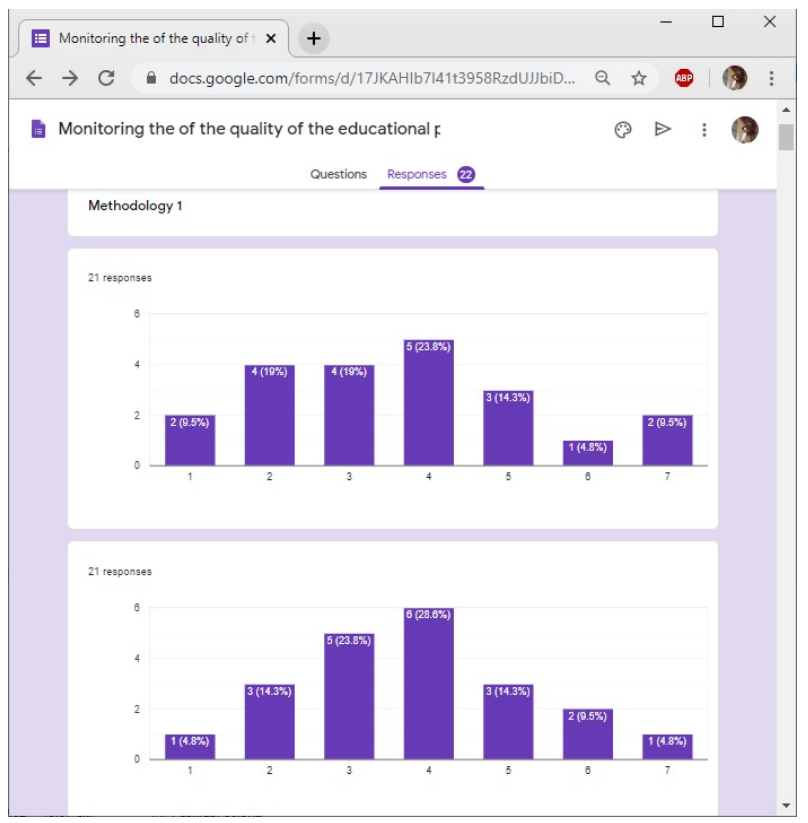

Fig. 4. The statistical analysis of the answers to the questions in the form of the linear scale.

The results of the study provide the following answers to the question - "yes, I would strongly like to move", "I do not know, it is hard to say", "would rather move than stay", "see no difference", "most likely would stay in to my unit", "would strongly like to stay in my 
unit".

The analysis of the results enables to make a deep analysis of the psychological conditions, as well as facilitate the development of recommendations, programs for improving the social and psychological climate in the educational institution.

Thus, in particular, the analysis of the results of the pilot survey of the Yuriy Fedkovych Chernivtsi National University teachers, the case of which is given in Fig. 5, allows to conclude about the significant problems of the psychological assurance of the quality of education, nevertheless, as it is seen from the figure, just $47,8 \%$ of teachers consider the relations in the team as favourable. $25 \%$ of respondents would change the jobs. It is quite problematic to provide a student-centred approach to the organization of the educational process in such situation. Accordingly, it is urgent to develop the special psychological and managerial measures to minimize the revealed negative tendencies in the activity and interaction of higher education institutions teachers.

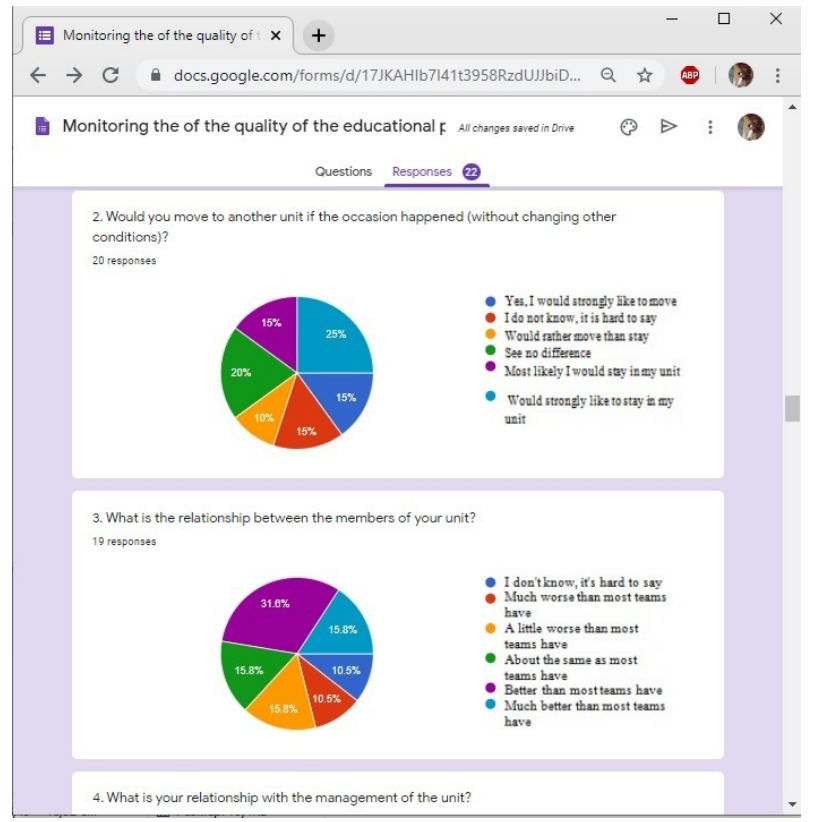

Fig. 5. The statistical analysis of the answers to the questions in the form of diagrams.

\section{Appraisal of Google Forms as a tool for monitoring the quality of education}

The monitoring of the quality of education with Google Form enables swiftly to get the feedback from the participants of the educational process on the quality and outcomes of the educational activity. This is evidenced, in particular, by the validation of the Google Forms created in the pilot study, which confirmed the efficiency in obtaining information, analysing the quality and percentage values in graphical and numerical format, reaching a large number of participants in a short time.

It is noteworthy, that the data from a Google Forms spreadsheet is easily imported into other spreadsheets (including SPSS) with aim of in-depth processing of results using factor, cluster or regression procedures and other kinds of statistical analysis.

Based on the evaluation of the monitoring results of the quality of education, a set of corrective and developmental measures is determined in accordance with the conceptual goals and objectives of the functioning and transformation of the educational system presently [21].

Consequently, the usage of Google services, the Google Form notably, contributes to the improvement of the internal system of the ensuring the monitoring of the quality of education, the development of science-based recommendations for the further improvement of the quality of education in higher education institutions with the psychological component, and furthermore, the improvement the competitiveness and attractiveness of educational institutions in regard to the analysis of their resources.

The documents and spreadsheets, created by using Google services, are stored on a cloud server (or can be saved to a file) [23], which is one of the key benefits of the program, since the access to the given data may be entered from any computer or mobile phone connected to the Internet.

Furthermore, the obtained resources can be used to adjust the management system in the educational institutions, managing goals, vision, mission, the educational environment [24], as well as to increase motivation and efficiency [25], to train a person to learn and apply modern international relations.

\section{Conclusions}

The authors described studies related to the efficiency monitoring improving of the quality of the psychological component of education through the implementation of information and communication technology to education, namely Google services, basing upon on the latest achievements and results of advanced research in the relevant fields. Google services opens up an extremely wide field of possibilities, enables to create text documents, presentations, spreadsheets, forms, drawings and other documents.

The monitoring organization of the quality of the psychological component of education with Google Form makes it possible not only to determine the nature of the relationship between the participants of the educational process and the level of satisfaction with them, the peculiarities of the socio-psychological climate as the level of development indicator of organizational culture, but also to make appropriate managerial decisions and forecast the situations in the educational environment; to promptly intervene and make appropriate adjustments to the educational process; to specifically plan the work on the relevant problem in higher education institutions; to create conditions for comparison of own assessment of pedagogical staff activity with independent assessment.

Thuswise, there is an urgent need to develop a reliable and valid toolkit for holistic and prompt monitoring of the quality of education using the Google services, not only on the psychological component, but 
also on all the criteria and indicators of the quality of higher education, which determines the prospects for further work.

\section{References}

1. World Education Forum, Incheon, Korea R (2015), http://unesdoc.unesco.org/images/0023/002338/233 813M.pdf. Accessed 21 Mar 2020

2. R.A. Madani, Analysis of Educational Quality, a Goal of Education for All Policy. Higher Education $\begin{array}{llll}\text { Studies } & 9(1), & 100-109 & \text { (2019). }\end{array}$ doi:10.5539/hes.v9n1p100

3. L. Schindler et al., Definitions of quality in higher education: A synthesis of the literature. Higher Learning Research Communications 5(3), 3-13 (2015). doi:10.18870/hlrc.v5i3.244

4. O. I. Bondarchuk, Subjective well-being of university teachers as a psychological factor of quality of higher education, in Management of higher education quality: problems and prospects, ed. by V. Oliynyk, pp. 86-89 (2017)

5. V.V. Balakhtar, The influence of information and communication technologies on the formation of professional competence of the personality of a social work specialist. Information Technologies and Learning Tools 66(4), 93-104 (2018)

6. A.V. Yakimenko, Google Drive service as an innovative means of cloud technologies. Young Scientist 5.3(57.3), 79-83 (2018)

7. W.D. Haddad, T. Demsky, Education PolicyPlanning Process: An Applied Framework. Fundamentals of Educational Planning 51 (UNESCO, Paris, 1995)

8. W. Glasser, The quality school: Managing students without coercion (Harper and Row Publishers, New York, 1990)

9. D. Adams, Defining educational quality, Improving Educational Quality, Project Publication 1: Biennial Report (1993)

10. T.I. Shamova, P.I. Tretyakov, N.I. Kapustin, Management Educational Systems (Vlados, Moscow, 2002)

11. V.A. Kalney, S.E. Shishova, School: monitoring the quality of education (Pedagogical Society of Russia, Moscow, 2000)

12. J.B. Babalola, Quality assurance and child friendly strategies for improving public school effectiveness and teacher performance in a democratic Nigeria, in Management of Primary and Secondary Education in Nigeria (NAEAP Publications, Ibadan, 2004), pp. 303-312

13. S. Babynets, Monitoring the quality of education: a pedagogical analysis (2020), https://osvita.ua/school $/$ method/353. Accessed 07 Feb 2020

14. V.V. Dron, Google services in teaching activity of teachers (2016), https://drive.google.com/file/d/0B6 y-TSh0wJSAVF84dkRWZllobkE/view. Accessed
28 Mar 2020

15. K. Azizova, Stages of monitoring the quality of education in higher education. The origins of pedagogical excellence 17, 3-12 (2016)

16. Regulations on monitoring the quality of educational activity and the quality of higher education of applicants (students, graduate students), improving the qualification of students at University of Educational Management, National Academy of Pedagogical Science of Ukraine (2017). http://umo.edu.ua/images/content/document/\%D0\% 9F\%D0\%BE\%D0\%BB $\%$ D0 $\%$ BE $\%$ D0 $\%$ B $6 \%$ D0 $\%$ B5\%D0\%BD $\%$ D0 $\%$ BD $\%$ D1\%8F\%20\%D0\%BF\%D $1 \% 80 \% \mathrm{D} 0 \% \mathrm{BE} \% 20 \% \mathrm{D} 0 \% 9 \mathrm{C} \% \mathrm{D} 0 \% \mathrm{BE} \% \mathrm{D} 0 \% \mathrm{BD}$ $\% \mathrm{D} 1 \% 96 \% \mathrm{D} 1 \% 82 \% \mathrm{D} 0 \% \mathrm{BE} \% \mathrm{D} 1 \% 80 \% \mathrm{D} 0 \% \mathrm{~B} 8 \%$ D0\%BD\%D0\%B3.pdf. Accessed 21 Mar 2020

17. V.Yu. Bykov, O.Yu. Kuchanskyi, A.O. Biloshchytskyi, Y.V. Andrashko, O.V. Dikhtiarenko, S.V. Budnik, Development of information technology of complex evaluation of higher education institutions activity. Information Technologies and Learning Tools 73(5), 293-306 (2019)

18. V.G. Kremen, V.Yu. Bykov, Categories of space and environment: peculiarities of model representation and educational application. Theory and practice of social systems management: philosophy, psychology, pedagogy, sociology 3, 316 (2013)

19. O.A. Gritsenyuk et al, The European Experience of Developing Digital Teacher Competence in the Context of Modern Educational Reforms. Information Technologies and Learning Tools 65(3), 316-336 (2018)

20. O.M. Spirin, T.A. Vakalyuk, Formation of Information and Communication Competence of Bachelors of Informatics on the Use of CloudOriented Learning Environment. Information Technologies and Learning Tools 72(4), 226-245 (2019)

21. N.P. Fetiskin, V.V. Kozlov, G.M. Manuilov, Sociopsychological diagnosis of personality development and small groups (Izdatelstvo Instituta Psikhoterapii, Moscow, 2005), pp. 190-199

22. M.A.R. Estrada, Policy modeling: Definition, classification and evaluation. J. of Policy Modeling 33(4), 523-536 (2011). doi:10.1016/j.jpolmod.2011.02.003

23. Y. Kondratenko, D. Simon, I. Atamanyuk, University Curricula Modification Based on Advancements in Information and Communication Technologies, in ICTERI 2016, pp. 184-199

24. T. Sranamkam, The effects of Web-based Instruction in physics entitle momentum using collaborative learning by Google application to enhance problem solving skills for grade XI students (2014). doi:10.2139/ssrn.2474698

25. T.O. Lukina, Monitoring as Information on Social Education. Testing and monitoring 12, 16-20 (2007) 